\title{
Impact Des Conflits Armes Sur La Sante Maternelle En Cote d'Ivoire: Cas Des Conflits Armes De 2002 Et De 2010
}

\author{
Djessou Flore Marie Hélèna Epse Tra, Doctorante \\ Université Félix Houphouët Boigny (FHB), Cocody-Abidjan, Côte d'Ivoire
}

Doi: 10.19044/esj.2018.v14n8p119 URL:http://dx.doi.org/10.19044/esj.2018.v14n8p119

\begin{abstract}
The "new conflict" that emerged in the 90s, mainly in developing countries, is a major obstacle to improving health. Quantitative studies of the impact of armed conflict on maternal health indicators are still fragmentary and scarce. The purpose of this study is to analyze the impact of the 2002 and the 2010 conflicts on maternal health in Côte d'Ivoire. In this study, we choose a particular type of health care service with assisted delivery in a health center. We use the parametric difference using the difference method to estimate our parameter of interest. Also, we use the propensity score technique and the standard error bootstrap technique to regulate some double difference hypotheses. In making our estimates, we use survey data including the MICS 2000 and 2006 and EDSCI 2012. Our results show that, globally, the crisis of 2002 had a negative effect on the use of assisted delivery in the CNO zone (Center North and West) $(-0,708)$. The same is true for the post-election crisis $(-0,514)$. On the other hand, in South and Abidjan zone, the 2002 crisis had no significant effect, while that of the 2010 crisis had a positive effect $(+0,628)$. The conflicts of 2002 and 2010 therefore constituted a major impediment to access to maternal health care services for households living in $\mathrm{CNO}$ zone.
\end{abstract}

Keywords: New conflicts, armed conflicts, maternal health, assisted delivery, Côte d'Ivoire

\section{Résumé}

Les «nouveaux conflits » apparus dans les années 90s principalement dans les pays en développement constituent un obstacle majeur à l'amélioration de la santé. Les études quantitatives consacrées à l'impact des conflits armés sur les indicateurs de santé maternelle demeurent encore fragmentaires et peu abondantes. L'objectif de cette étude est d'analyser l'impact du conflit de 2002 et celui de 2010 sur la santé maternelle en Côte d'Ivoire. Nous choisissons un type particulier de service de soins de santé, 
l'accouchement assisté dans un centre de santé. Nous utilisons la méthode paramétrique de double différence pour estimer notre paramètre d'intérêt. Nous utilisons également la technique du score de propension et celle du bootstrap de l'erreur standard en vue de relâcher certaines hypothèses de double différence. Pour effectuer nos estimations, nous mobilisons les données d'enquêtes notamment les MICS 2000 et 2006 et l'EDSCI 2012. Nos résultats montrent que de façon globale la crise de 2002 a eu un effet négatif sur le recours à l'accouchement assisté en zone CNO (Centre Nord et Ouest) $(-0,708)$. Il en est de même pour la crise post-électorale $(-0,514)$. Par contre dans la zone Sud et Abidjan la crise de 2002 n'a pas eu d'effet significatif et celle de 2010 a exercé un effet positif $(+0,628)$. Les conflits de 2002 et de 2010 ont donc constitué une entrave majeure à l'accès aux services de soins de santé maternelle pour les ménages vivant en zone CNO.

Mots-clés : Conflits armés, santé maternelle, accouchement assisté, Côte d'Ivoire

\section{Introduction}

Durant ces dernières années, les questions rattachées à la santé semblent davantage préoccuper la communauté internationale. L'amélioration de la santé constitue un axe essentiel de l'action en faveur du développement. C'est dans cette optique que 189 nations présentes au sommet du Millénaire en 2000 ont adopté la déclaration du Millénaire des Nations unies qui définit les objectifs à atteindre par la communauté internationale jusqu'à 2015. Trois axes ont été consacrés à l'amélioration de la santé à savoir : l'OMD 4 qui vise à réduire la mortalité de l'enfant; l'OMD 5 qui vise à améliorer la santé maternelle et l'OMD 6 qui vise à combattre le VIH/SIDA, le paludisme et d'autres maladies.

Au regard de la gravité de la situation maternelle dans la plupart des pays africains notamment ceux au Sud du Sahara, les dirigeants africains ont choisi de se focaliser sur l'OMD 5 «Améliorer la santé maternelle» avec ses deux cibles, à savoir «Réduire de trois quarts, entre 1990 et 2015, le taux de mortalité maternelle: cible 5A » et "Rendre l'accès à la médecine procréative universel jusqu'à 2015 : cible $5 B$ ». Par ce choix, ils accordent une priorité à sauver la vie des femmes, mais également à accroître leur bienêtre économique et social, améliorer la vie de leurs enfants et de leur famille et réduire la pauvreté. Par ailleurs les Objectifs de Développement Durable (ODD) 2015-2030 qui succèdent aux OMD définissent une nouvelle initiative de transformation de la santé maternelle, visant à mettre un terme à la mortalité maternelle évitable ; la cible 3.1 de l'objectif ODD 3 est de faire passer le taux mondial de mortalité maternelle en-dessous de 70 pour 100000 naissances vivantes d'ici à 2030 . 
Malgré les efforts consentis par ces pays en adoptant ces différentes déclarations, le bilan est peu reluisant. Selon l'OMS, en 2013 le taux de mortalité maternelle dans les régions en développement était près de 14 fois supérieur à celui des régions développées.

Le rapport de Save the children (2014) fait également ressortir que les indicateurs de santé maternelle sont très bas en Afrique. Save the children après avoir évalué le bien-être des mères et des enfants dans 178 pays a procédé à un classement. Parmi les 178 pays évalués, les pays africains se retrouvent au bas du palmarès en occupant les 10 dernières places. La quasitotalité de ces pays sont des pays d'Afrique Centrale, d'Afrique de 1'Est et de l'Ouest. Ce sont notamment par ordre décroissant (178ème au 169ème), la Somalie, la République Démocratique du Congo, le Niger, le Mali, la GuinéeBissau, la République Centrafricaine, la Sierra-Leone, le Nigeria, le Tchad et la Côte d'Ivoire. Tous les pays figurant parmi les 10 derniers ont des antécédents de conflit armé. Selon la Banque Mondiale, les pays en conflit se situent à $14 \%$ pour l'OMD 5 , or l'objectif est d'atteindre $75 \%$. En effet, un épisode même bref de conflit armé peut interrompre des progrès et effacer des gains obtenus au fil des générations, compromettant l'accès aux services sociaux de base notamment les services de soins de santé maternelle. L'impact des conflits est d'autant plus grave et prolongé quand les pays ont une structure de l'économie qui ne permet pas de résister et de rebondir rapidement comme c'est le cas pour la plupart des pays en développement.

Si nous prenons un pays comme la Côte d'Ivoire, ce pays d'Afrique de l'Ouest jadis connu pour sa longue stabilité politique a souffert de conflits de plus en plus violents, à commencer par un coup d'Etat vers la fin de l'année 1999, qui a précédé un conflit armé à part entière en 2002 pour déboucher sur le conflit post-électoral de 2010. Les tendances de la mortalité maternelle sur la période allant de 1990 à 2015 pour ce pays indiquent qu'il n'y a pas eu de progrès vers la réalisation de l'OMD 5A (OMS, 2015). Le Rapport de Mortalité Maternelle (RMM, décès pour 100000 naissances vivantes) est passé de 745 en 1990 ; à 711 en 1995 ; puis 671 en $2000 ; 742$ en $2005 ; 717$ en 2010 et 645 en 2015. Au cours de cette même période la Côte d'Ivoire a connu des situations d'instabilité et cela aurait certainement contrarié fortement les efforts de réduction de la mortalité maternelle. Dans de telles situations, l'effondrement des systèmes de santé peut provoquer la montée en flèche des décès résultant des complications qui auraient été aisément traitées dans des conditions stables.

Ce constat nous emmène à tester à partir d'un modèle économétrique l'impact que les conflits de 2002 et de 2010 ont eu sur la santé maternelle en Côte d'Ivoire, en nous intéressant à un type particulier de soins de santé : l'accouchement assisté dans un centre de santé. Pour parvenir à nos résultats, nous mobilisons les données d'enquête ménage notamment les MICS 
(Enquête par grappe à indicateurs multiples) 2000 et 2006 et l'EDSCI (Enquête démographique et de santé) 2012.

Le reste de l'article est organisé comme suit. La section 2 présente la revue de littérature sur les conséquences des conflits armés sur la santé maternelle. La section 3 décrit le déroulement des conflits. La section 4 traite de la stratégie d'identification utilisée dans cet article, fournit des informations sur les données, les variables et la constitution des groupes. La section 5 présente les résultats et discussion et la section 6 donne la conclusion.

\section{Revue de Littérature}

Dans le champ de l'économie, les premières études établissant le lien entre conflits armés et indicateurs du développement humain se sont penchées sur les causes des conflits. Elles ont porté sur les griefs socio-économiques, politiques et culturels pouvant conduire à un mécontentement généralisé et activé la rébellion (Kaplan, 1994 ; Rice, 2006). Certains auteurs tels que Hugon (2001) et Cartier (2010) ont mis l'accent sur les facteurs qui créent et exacerbent les griefs, ce sont notamment les modèles historiques de développement, le rôle de l'Etat et les politiques internationales. En revanche des auteurs tels que Collier et Hoeffler (1998), Fearon et Laitin (2003), ont expliqué l'apparition des conflits par la cupidité des rebelles. Selon eux, les pays riches en ressources naturelles ont un risque plus élevé de conflit.

Plus récemment, l'attention s'est tournée vers les conséquences de la guerre (Justino, 2012 ; Yabilé, 2013 ; Garcia \& Bartlett, 2015 ; Palmer et al., 2016; Alkresh et al., 2016). Avec la disponibilité croissante de données fournies d'une part par les organismes internationaux et d'autre part, par les enquêtes sur les ménages dans les pays en développement, les chercheurs se sont penchés sur l'impact des conflits armés sur les indicateurs du développement humain notamment dans le domaine de l'éducation, de la santé et du bien-être des ménages.

Cependant, les études consacrées essentiellement à l'impact des conflits armés sur les indicateurs de santé maternelle demeurent encore fragmentaires et peu abondantes.

\section{Concernant Les Soins Prénatals}

Price et Bohara (2013), en utilisant les données d'enquêtes auprès des ménages, ont examiné l'effet des conflits armés sur l'utilisation des soins prénatals au Népal. Selon eux, il existe une corrélation négative entre le nombre de visites de soins prénatals et les incidences de violence liées au conflit dans les zones touchées. Ils notent également que les femmes vivant dans les zones de conflit à haute intensité reçoivent entre 0,3 et 1,5 fois moins de soins que leurs homologues vivant dans les zones moins affectées. Ils tirent 
la conclusion selon laquelle l'utilisation des soins de santé prénatals est en partie déterminée par l'environnement socio-politique.

S’agissant de La Mortalité Maternelle, O Hare et Southall (2007), ont choisi 42 pays d'Afrique Subsaharienne dont 21 exposés aux conflits armés et 21 non exposés aux conflits. Ils ont comparé ces deux groupes en termes de taux de mortalité maternelle. Leurs résultats montrent que le taux de mortalité dans les pays en conflit est en moyenne de 1000 décès pour 100000 naissances vivantes contre 690 décès pour 100000 naissances vivantes dans les pays sans conflit. Le taux de mortalité est également significativement plus élevé dans les pays ayant connu un conflit récent.

L'effet négatif des conflits armés sur la mortalité maternelle apparaît également dans le rapport 2015 de l'OMS portant sur les tendances de la mortalité maternelle, 1990-2015. Si nous prenons les dix derniers pays cité précédemment, les résultats des estimations du Rapport de Mortalité Maternelle (RMM ; décès pour 100000 naissances vivantes) sont insatisfaisants. Le RMM est de $2 \%$ en Somalie ; $21,2 \%$ en République Démocratique du Congo; 36,7\% au Niger; 41,9\% au Mali ; 39,5\% en Guinée-Bissau ; 48,3\% en Sierra Leone ; 41,0\% au Tchad ; le rapport conclue que les progrès effectués dans ces pays sont insuffisants. Le RMM est de $31,6 \%$ en République Centrafricaine; 39,7\% au Nigeria; $13,4 \%$ en Côte d'Ivoire ; selon ce rapport il n'y a pas eu de progrès dans ces pays. Comme nous pouvons le constater, aucun de ces 10 pays ayant eu un antécédent de guerre n'a pu atteindre la Cible 5.A qui est de réduire de trois quarts $(75 \%)$ jusqu'à 2015, le taux de mortalité maternelle.

Concernant L'Accouchement Assisté Dans Un Centre de Santé : Gudrun et al. (2015) ont montré que les conflits armés ont un effet néfaste sur l'accès aux soins de santé maternelle et reproductive, en particulier le recours à l'accouchement assisté dans un centre de santé. Pour examiner cette question, ils ont utilisé les données d'enquêtes démographiques et de santé dans 31 pays d'Afrique subsaharienne entre 1990 et 2013 portant sur les mères âgées de 15 à 45ans. En vue d'effectuer leurs estimations, ils ont utilisé le modèle à effets fixes. Leurs résultats indiquent que l'intensité des conflits réduit considérablement la probabilité qu'une femme donne naissance dans un établissement médical. Cet impact négatif est plus accentué dans les zones urbaines bien que le niveau de soins maternels soit plus faible dans les zones rurales. En outre, les femmes pauvres, moins instruites sont également fortement touchées par les conflits en matière d'assistance médicale pendant l'accouchement.

En Côte d'Ivoire, les travaux établissant un lien entre conflits armés et santé, se sont pour la plupart penchés sur l'impact des conflits armés sur le système de santé et sur la santé infantile (Tiembre et al., 2011 ; Minoiu \& Shemyakina, 2014; Idrissa Ouili, 2014). Par ailleurs elles se sont plus 
focalisées sur le conflit armé de 2002. De façon générale, les études qui se sont intéressées au conflit armé de 2010 sont des rapports d'évaluation commandités par les Etats et les institutions (Banque Mondiale, le ministère de la santé et de la lutte contre le VIH, UNFPA, etc.).

Selon les Fonds des Nations Unies pour la Population (UNFPA), la Côte d'Ivoire enregistre des taux de mortalité maternelle élevés (OMS, 2015). Chaque jour, ce sont plus de 16 femmes qui meurent de complications liées à la grossesse ou à l'accouchement, le plus souvent du fait de problèmes médicaux pouvant être soignés (EDSCI, 2012). Ces décès maternels sont essentiellement dus à des causes médicales directes dont les plus fréquentes sont les hémorragies ante et post-partum (41\%), les grossesses extra-utérines (18\%) (Enquête Santé Mère \& Enfant, 2010). Contrairement aux prévisions faites, le taux de mortalité maternelle a connu une hausse : ce ratio est passé de 543 décès maternels pour 100000 naissances vivantes en 1994 à 614 décès maternels pour 100000 naissances vivantes en 2012. Cependant, ces rapports ne font aucune analyse causale de l'impact des conflits armés sur la santé maternelle et particulièrement sur la probabilité d'être assisté à l'accouchement dans un centre de santé.

Devant ces insuffisances une question nous vient à l'esprit : existe-t-il une relation de cause à effet entre conflit armé et santé maternelle en Côte d'Ivoire ? De façon spécifique : quel est l'effet causal des conflits de 2002 et de 2010 sur le recours à l'accouchement assisté en Côte d'Ivoire?

Notre analyse permettra d'évaluer pour la première fois en Côte d'Ivoire, l'effet causal des conflits de 2002 et de 2010 sur le recours à l'accouchement assisté dans un centre de santé. Par conséquent une telle étude permettra de mieux cibler les femmes effectivement affectées par les conflits et mener une action ciblée en leur faveur.

\section{Déroulement des Confits en Côte d'Ivoire}

La Côte d'Ivoire, après trente ans de stabilité politique va connaître des situations de violence de plus en plus grave. Après la mort du premier président Félix Houphouët Boigny, le président Henri Konan Bédié accède au pouvoir en 1993. En 1995, il met sur pied le concept de «l'ivoirité», établissant une distinction entre les ivoiriens de souche et les étrangers. Cette politique écarte donc de l'élection présidentielle l'ancien premier ministre Alassane Ouattara, devenu un opposant politique. Cette décision suscite la colère de l'opposition.

Par la suite, Le 24 décembre 1999, une vague de mécontentement chez des militaires est à l'origine d'un coup d'Etat qui permet à l'ancien général Gueï Robert de renverser le président Henri Konan Bédié et de prendre le contrôle du pays. Un Comité National de Salut Public (CNSP) est formé et Gueï fait la promesse de respecter le système démocratique en tenant des 
élections le plus tôt possible. Pour sa part, le président déchu fuira vers le Togo, puis vers la France. Le général Gueï tiendra sa promesse et une élection présidentielle aura lieu en Côte d'Ivoire le 26 octobre 2000. La contestation de la victoire du président élu va entraîner des troubles à Abidjan. Le président sortant s'inclinera finalement devant Laurent Gbagbo du Front Populaire Ivoirien (Perspective Monde, 1999).

Le 19 septembre 2002, des soldats et des rebelles du mouvement patriotique de Côte d'Ivoire (MPCI) tentent de contrôler les villes d'Abidjan, Bouaké et Korhogo, alors que le président Gbagbo se trouve à l'extérieur du pays (Italie). Les motivations sont des griefs qui remontent à 1995 au sujet du concept de «l'ivoirité ». Aussi, lorsque les rebelles lancent leur attaque le 19 septembre 2002, le pays est littéralement divisé en deux, le Sud sous contrôle gouvernemental et les zones Centre, Nord et Ouest (CNO) sous contrôle des Forces Nouvelles (Perspective Monde, 2002). Pour tenter de résoudre cette crise, plusieurs accords (accords d'Accra, accords de Linas Marcoussis, accords de Pretoria) vont être signés entre les différents belligérants. Mais ceux-ci ne suffiront pas pour calmer les ardeurs des uns et des autres. C'est seulement en mars 2007 avec la signature de l'Accord politique de Ouagadougou (Burkina Faso) que le processus de paix semble être enclenché avec pour point focal, l'organisation d'élections libres, transparentes et ouvertes à tous. Les populations peuvent désormais circuler sans entraves majeures sur l'ensemble du territoire national. Au cours de l'année 2008, l'environnement sécuritaire était relativement stable en dépit de quelques remous sporadiques dans certaines villes.

Tous les accords signés depuis le début de la crise, dont le dernier en date notamment l'Accord de Paix de Ouagadougou (APO) ont préconisé un désarmement préalable aux élections générales. Malgré tout, d'un commun accord des deux bords, le scrutin présidentiel du 31 octobre 2010 s'est tenu sans que cette volonté n'ait été réalisée. La crise post-électorale est une crise politique qui débute après le second tour de l'élection présidentielle ivoirienne qui s'est tenu le 28 novembre 2010, le premier tour s'étant tenu le 31 octobre 2010. Ce scrutin s'est soldé par un différend électoral. Les deux candidats, Laurent Gbagbo, président sortant et reconnu par le Conseil constitutionnel, et Alassane Ouattara, reconnu par la Commission électorale indépendante et la communauté internationale, revendiquent chacun la victoire. Les résultats contestés de l'élection présidentielle relancent les hostilités.

Ces deux conflits armés ont engendré de lourdes conséquences non seulement sur le plan humain (des milliers de morts) mais surtout sur le plan socio-économique. Il s'agit de nombreux mouvements internes et externes de population. Des exactions et violations flagrantes des droits de l'homme. Ils ont remis en cause les fondements de l'unité nationale et perturbé le fonctionnement normal des institutions, avec pour conséquences, l'effritement 
de la cohésion sociale, le ralentissement des activités économiques et financières (fermeture des entreprises, licenciement massif), la dégradation des services sociaux tels que ceux de la santé maternelle.

\section{Stratégie D'identification}

Nous cherchons à établir un lien de cause à effet entre conflit et la variable de résultat : recours à l'accouchement assisté dans un centre de santé.

\section{Cadre Conceptuel de Rubin (1974)}

Nous nous situons dans le cadre conceptuel de Rubin (1974). Selon Rubin, on mesure la causalité en utilisant la notion de contrefactuel. Il indique une situation virtuelle qui se serait produite si le choc (ici le conflit armé) dont on cherche à mesurer l'impact n'avait pas eu lieu. L'écart entre la réalité et le contrefactuel correspond à l'impact causal.

$$
\Delta_{i}=Y_{i 1}-Y_{i 0}
$$

Le contraste est qu'on ne peut pas observer simultanément les deux situations dans lesquelles le même individu aurait été «traité » et «non traité ». Rubin construit donc un contrefactuel qui représente cette situation hypothétique. Le terme traitement fait référence aux sciences médicales dans laquelle on analyse l'effet d'un traitement sur un ensemble d'individus. Aujourd'hui, il est employé dans les sciences sociales pour désigner une intervention ou une politique que l'on cherche à évaluer.

\section{Les Deux Grandes Approches Utilisées Pour Construire le Contrefactuel}

Les deux grandes approches couramment utilisées pour construire le contrefactuel sont l'approche expérimentale encore appelée approche randomisée et l'approche quasi-expérimentale.

Le principe de l'approche expérimentale est de s'approcher au mieux des essais cliniques. La règle générale est celle de l'assignation aléatoire. Dans cette approche, le chercheur crée de manière aléatoire le groupe de contrôle (contrefactuel). Selon Ahouré et Ouattara (2009), dans le cas de l'approche expérimentale, l'hypothèse d'indépendance est satisfaite et la variable de traitement est dite exogène.

$$
\left(Y_{i 1}, Y_{i 0}\right) \perp T_{i}
$$

Selon Antonakis et al. (2010), cette approche est l'étalon or. Cependant, elle pose des problèmes d'éthiques parce qu'il s'agit d'expériences faites directement sur des individus.

Quant à l'approche quasi-expérimentale encore appelée approche non expérimentale, le principe général est d'utiliser des techniques statistiques en se basant sur des données d'observation (enquête) pour créer le groupe de contrôle (Cadot et al., 2014). La règle est celle de l'assignation non aléatoire. 
La difficulté à laquelle est confrontée le chercheur est de trouver un contrefactuel crédible.

Dans la littérature, plusieurs méthodes à la fiabilité éprouvée ont été proposées. Elles sont toutes associées à un ensemble d'hypothèses.

\section{Les Méthodes Quasi-expérimentales Couramment Utilisées}

Les méthodes quasi-expérimentales couramment utilisées sont: la méthode des variables instrumentales (Angrist et al., 1996), la régression par discontinuité (Givord, 2010), l'appariement sur score de propension (Rosenbaum \& Rubin, 1983) et la double différence (Imbens \& Wooldridge ,2009). La difficulté au niveau des variables instrumentales est que les meilleurs instruments sont ceux fournis dans le cadre de la randomisation (Givord, 2010), or nous nous situons dans un cadre non expérimental. Quant à la régression par discontinuité, la difficulté réside dans le choix d'un seuil crédible.

\section{Stratégie D’identification par Double Différence}

Nous avons opté pour la méthode de double différence et le principe de cette méthode est résumé dans le Tableau1.

Tableau 1. Présentation de la méthode de double différence

$\begin{array}{cccc} & \text { Pré-conflit } & \text { Post-conflit } & \text { Changement } \\ \text { Groupe 1(traité) } & Y_{10} & Y_{11} & \Delta Y_{T}=\left(Y_{11}-Y_{10}\right) \\ \text { Groupe 2 } & Y_{20} & Y_{21} & \Delta Y_{c}=\left(Y_{21}-Y_{20}\right) \\ \text { (Contrôle) } & & & \text { DID }=\Delta Y_{T}-\Delta Y_{c} \\ \text { DID } & & & \end{array}$

- $\quad$ Principe de la méthode de double différence

Il s'agit de constituer deux groupes : un groupe traité et un groupe contrôle (contrefactuel) et d'avoir deux périodes : une période pré-conflit et une période post-conflit. On calcule une première différence pour les traités avant et après conflit et une deuxième différence pour les contrôles avant et après conflit et ensuite on calcule la différence des différences.

- $\quad$ Estimateur de la double différence par la méthode paramétrique

Nous choisissons d'estimer la double différence par la méthode paramétrique. Notre variable de résultat étant binaire, nous optons pour le modèle Logit qui est un modèle qui peut être étendu à la double différence en ajoutant des termes d'interaction (Mandic, 2012). En nous inspirant du modèle de double différence d'Audibert et al. (2015), nous spécifions notre modèle comme suit :

avec

$$
Y_{i t}=\beta_{0}+\beta_{1} T_{i}+\tau t_{1}+\delta\left(T_{i} * t_{1}\right)+\gamma X_{i t}+\varepsilon_{i t}
$$

$\boldsymbol{Y}_{i t}$ : la variable de résultat de l'individu $i$ à la période $t$. 
$\boldsymbol{T}_{\boldsymbol{i}}$ : la variable indiquant le statut de traitement, qui prend la valeur 1 si l'individu se trouve

en zone de conflit et 0 sinon.

$\boldsymbol{t} \quad$ : la variable temporelle, égale à 0 pour l'année avant conflit et 1 pour l'année post-conflit.

$\boldsymbol{T}_{\boldsymbol{i}} * \boldsymbol{t}$ : le terme d'interaction des deux variables.

$\boldsymbol{X}_{i t}$ : représente les caractéristiques associées à l'individu.

$\boldsymbol{\varepsilon}_{\boldsymbol{i t}} \quad:$ le terme d'erreur.

$\boldsymbol{\delta}$ est l'estimateur de double différence, il mesure l' effet moyen du conflit.

$$
\begin{gathered}
\delta=D I D=\Delta Y_{T}-\Delta Y_{c} \\
=\left(Y_{11}-Y_{10}\right)-\left(Y_{21}-Y_{20}\right)
\end{gathered}
$$

- Hypothèses de double différence

La méthode de double différence repose sur deux hypothèses fondamentales à savoir: L'hypothèse de tendance temporelle commune et l'hypothèse d'exogénéité.

Il est souvent difficile de valider l'hypothèse de tendance temporelle commune. Si les caractéristiques observables ne sont pas similaires, les tendances pourraient différer de manière non prévisible. Une alternative qui s'offre au chercheur est d'utiliser le score de propension. Cependant, avec cette technique, nous ne pouvons pas contrôler les caractéristiques inobservables. Nous introduisons donc des variables de contrôle enfin de réduire tout biais qui serait susceptible de surestimer ou sous-estimer nos estimations.

L'hypothèse d'exogénéité peut être également vérifiée par la technique du score de propension. Selon Ahouré et Ouattara (2009), dans le cas des études quasi-expérimentales, l'hypothèse d'indépendance n'est pas satisfaite, on doit faire l'hypothèse d'indépendance conditionnelle :

$$
\left.\left(Y_{i 1}, Y_{i 0}\right) \perp T_{i} \mid X_{i}\right)
$$

Si l'hypothèse d'indépendance conditionnelle est satisfaite, la variable de traitement est dite exogène et on peut appliquer la méthode de double différence. Le score de propension permet donc de relâcher les hypothèses de double différence (Marbot, 2010).

\section{La Technique du Score de Propension}

Le score de propension a été introduit en 1983 par Rosembaum et Rubin. Il désigne la probabilité d'être exposé au traitement selon un ensemble de caractéristiques observables (situation matrimoniale, sexe, niveau d'éducation etc...). L'expression du score est la suivante : $\boldsymbol{e}\left(\boldsymbol{X}_{\boldsymbol{i}}\right)=\boldsymbol{p}\left(\boldsymbol{T}_{\boldsymbol{i}}=\right.$ $\left.\boldsymbol{1} \mid \boldsymbol{X}_{\boldsymbol{i}}\right)$ avec $\mathbf{0}<\boldsymbol{e}\left(\boldsymbol{X}_{\boldsymbol{i}}\right)<\mathbf{1}$

Il s'agit pour nous de construire un groupe contrôle (les ménages n'ayant pas été touchés par le conflit), proche du groupe traité (c'est-à-dire les ménages ayant été touchés par le conflit) en terme de caractéristiques observables (score 
de propension). Nous calculons le score de propension à l'aide du modèle Logit. Le score de propension repose également sur deux hypothèses fondamentales à savoir: l'hypothèse d'indépendance conditionnelle et l'hypothèse de support commun. Nous vérifions l'hypothèse d'indépendance conditionnelle à l'aide du test d'équilibre de Becker et Ichino (2002) c'est-àdire le balancing test et nous vérifions l'hypothèse de support commun de façon visuelle à travers les graphes.

\section{Données et Constitution des Groupes}

Pour parvenir à nos résultats, nous utilisons les données d'enquête notamment les enquêtes par grappes à indicateurs multiples (Multiple Indicator Cluster Survey-MICS 2000 et 2006) et l'enquête démographique et de santé (EDSCI 2012). Notre intérêt pour ces types d'informations s'explique par le fait que pour calculer l'estimateur de double différence nous avons besoin de données avant et après conflit.

La deuxième enquête par grappe à indicateurs multiples connu sous le vocable anglais de Multiple Indicator Cluster Survey (MICS 2000) a été réalisée par l'UNICEF en collaboration avec l'OMS, l'UNESCO et d'autres agences des Nations Unies. Elle s'est déroulée du 8 juin au 8 août 2000 dans un environnement caractérisé par la transition politique suite au coup d'état du 24 décembre 1999. L'enquête a permis de disposer de données actualisées nécessaires qui ont servi à l'établissement du rapport de fin de décennie du Sommet Mondial pour les enfants (SME). Elle a porté sur un échantillon de 7 333 ménages. La portée de l'Enquête par grappe à Indicateurs Multiples (MICS 2000) comprend un questionnaire Ménage, un questionnaire Femme et un questionnaire Enfant.

L'enquête par grappe à indicateurs multiples, cycle 3 (MICS 2006) est le troisième cycle des enquêtes MICS. Les cycles 1 et 2 ont été réalisés respectivement en 1995 (MICS 1995) et 2000 (MICS 2000). Bien qu'un certain nombre de changements aient été faits dans la définition de certains indicateurs entre les différents cycles, de nombreuses questions et indicateurs de MICS (2006) sont compatibles avec ceux du cycle précédant (MICS 2000). Cette enquête a été réalisée par l'Institut National de la Statistique (INS). Outre la contribution du Gouvernement, une assistance technique et financière a été fournie par le Fonds de Nations Unies pour l'Enfance (UNICEF) et d'autres organismes internationaux. Elle s'est réalisée dans un contexte national marqué par la grave crise militaro-politique qu'a connu le pays depuis septembre 2002, avec la moitié nord hors de contrôle du Gouvernement. Elle a porté sur un échantillon de 7600 ménages.

La troisième enquête démographique et de santé (EDSCI-III), combinée à l'Enquête par grappe à Indicateurs Multiples (MICS) a été réalisée de décembre 2011 à mai 2012 par le Ministère de la Santé et de la Lutte contre 
le Sida (MSLS) en collaboration avec l'Institut National de la Statistique (INS). L'EDS-MICS 2011-2012 a bénéficié de l'assistance technique du programme mondial des Enquêtes Démographiques et de Santé (Demographic and Health Surveys-MEASURE DHS) d'ICF International. L'enquête a été financée par l'Etat de Côte d'Ivoire, l'Agence des Etats-Unis pour le Développement International (USAID), l'UNICEF, L'UNFPA, l'Union Européenne, la Banque Mondiale, le Fonds Mondial et l'ONUSIDA. L'enquête a porté sur un échantillon national de 10.413 ménages. Tout comme les MICS 2000 et 2006 l'échantillon de l'enquête EDSCI (2012) a été conçu afin de fournir des estimations sur les indicateurs de santé au niveau national, pour les zones urbaines et rurales, et pour les dix régions ci-après : le Centre, le Centre-nord, le Nord-est, le Centre-est, le Sud (n'incluant pas la ville d'Abidjan), le Sud-ouest, le Centre-ouest, l'Ouest, le Nord-ouest et le Nord correspondant à une strate. En raison de sa spécificité (capitale économique et grande mégalopole représentant près du cinquième de la population totale du pays au recensement de 1998), la ville d'Abidjan a été considérée comme une strate à part entière.

En vue d'identifier les groupes traités et contrôles, nous nous servons des données Armed Conflit Location \& Event Data Project (ACLED) et la carte de partition de la Côte d'Ivoire. Les données ACLED sont une collection des données publiques sur la violence politique pour les Etats en développement. Ces données contiennent des informations sur les dates et lieux de la violence, les types d'événements, les groupes impliqués, les décès et les changements dans le contrôle du territoire. Les informations sont enregistrées sur les batailles, les meurtres, les émeutes, etc... Ainsi, nous avons constitué les groupes suivants :

Tableau 2. Constitution des groupes traité et contrôle

\begin{tabular}{|c|c|c|}
\hline Groupe & Conflit de 2002 & Conflit de 2010 \\
\hline Traité & $\begin{array}{l}\text { Centre-nord } \\
\& \text { Ouest }(\mathrm{CNO})\end{array}$ & $\begin{array}{l}\text { Bouaké, Dabakala, Béoumi, Sakassou, } \\
\text { Katiola, Niakaramandougou, Man, Danané, } \\
\text { Biankouma, Kouibly, Zouhan Hounien, } \\
\text { Bangolo, Duekoué, Guiglo, Blolequin et } \\
\text { Toulepleu. }\end{array}$ \\
\hline
\end{tabular}

Contrôle Sud \& Abidjan Abidjan, Grand-Lahou, Tiassalé, Alépé, Dabou, Jacqueville, Sikensi, Adzopé, Agboville, Akoupé, Divo, Lakota, Gueyo, Aboisso, Adiaké, Grand-Bassam et Tiapoum.

Abengourou, Agnibilékrou et Bettié. Contrôle: Centre-est

Source : compilation de l'auteur à partir du DSRP ${ }^{24}, 2008$

${ }^{24}$ DSRP : Document de Stratégie pour la Réduction de la Pauvreté 
A l'aide des échantillons constitués, nous avons effectué le double appariement et nous avons obtenu les graphes suivants :

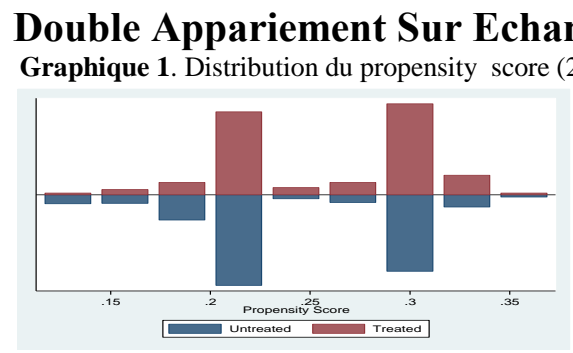

Source : calcul de l'auteur (MICS 2000)

Graphique 3. Distribution du propensity score (2006)

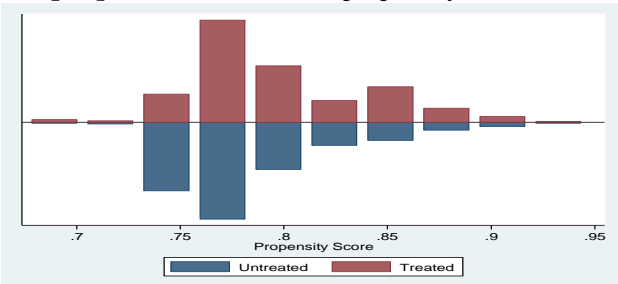

Source : calcul de l'auteur (MICS 2006)
Réduit au Support Commun

Graphique 2. Distribution du propensity score (2006)

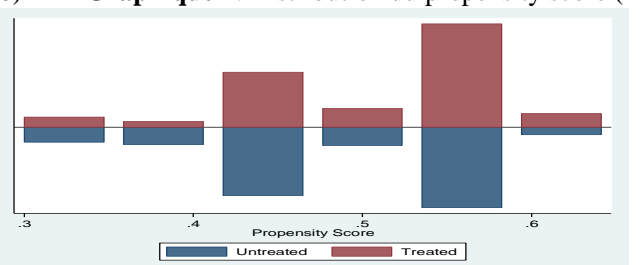

Source : calcul de l'auteur (MICS 2006)

Graphique 4. Distribution du propensity score (2012)

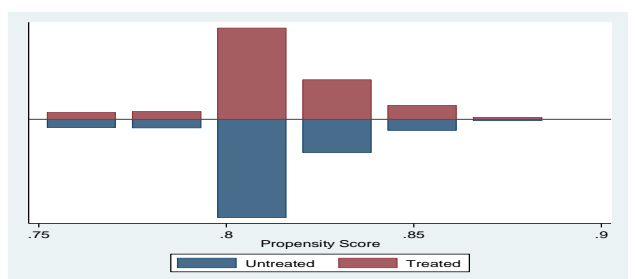

Source : calcul de l'auteur (MICS 2012)

Les graphes obtenus montrent que les groupes traités et contrôles sont équilibrés. Ils sont semblables sur la base de leurs caractéristiques observables. Nous avons retenons l'échantillon réduit au support commun pour effectuer nos estimations en double différence. Soulignons que les méthodes classiques d'inférence statistique ne permettent pas d'obtenir des réponses correctes à tous les problèmes que se pose le chercheur. Dans le cas où certaines conditions d'application ne sont pas remplies, une alternative qui s'offre au chercheur est l'utilisation du bootstrap qui est une méthode non paramétrique. Ce qui a fait dire à Rapacchi (1994) qu'avec le bootstrap, on est libre des hypothèses. Nous avons donc effectué le bootstrap de l'erreur standard et nous avons obtenu les résultats suivants.

\section{Résultats et Discussion}

Nous présentons les résultats de double différence sur score de propension pour les deux crises.

\section{Résultats et Discussion Pour le Conflit de 2002}

Le Tableau 3 présente les résultats de l'effet moyen du conflit de 2002 sur le recours à l'accouchement assisté des ménages vivant en zone CNO. 
Tableau 3. Effet moyen du conflit de 2002 sur le recours à l'accouchement assisté

\begin{tabular}{|c|c|c|c|c|}
\hline Variables & \multirow[b]{2}{*}{ Coef. } & Bootstrap & \multirow[b]{2}{*}{$\rho>|Z|$} & \multirow[b]{2}{*}{ Effets } \\
\hline Accou_assis & & Std.Err & & \\
\hline & \multicolumn{4}{|c|}{ Sans variables de contrôle } \\
\hline \multirow[t]{2}{*}{ Traité* year } & -1.064 & 0.125 & $0.000 * * *$ & - \\
\hline & \multicolumn{4}{|c|}{ Avec variables de contrôle } \\
\hline Traité*year & -0.708 & 0.115 & $0.000 * * *$ & - \\
\hline year_2006 & 0.047 & 0.106 & 0.657 & \\
\hline $\begin{array}{c}\text { Sexe_chef (ref. } \\
\text { male) }\end{array}$ & -0.139 & 0.154 & 0.365 & \\
\hline $\begin{array}{l}\text { Age_chef } \\
\text { (ref.adulte) }\end{array}$ & -0.126 & 0.112 & 0.262 & \\
\hline \multicolumn{5}{|c|}{ Education (ref.sans educ) } \\
\hline Primaire & 0.680 & 0.113 & $0.000 * * *$ & + \\
\hline Secondaire et plus & 1.352 & 0.187 & $0.000 * * *$ & + \\
\hline \multicolumn{5}{|c|}{ Religion (ref. catholique) } \\
\hline Protestant & -0.258 & 0.153 & $0.092 *$ & + \\
\hline Musulman & 0.466 & 0.146 & $0.001 * * *$ & + \\
\hline Autre religion & -1.110 & 0.152 & $0.000 * * *$ & - \\
\hline \multicolumn{5}{|l|}{ Constante } \\
\hline Observations & & & & 2234 \\
\hline Pseudo $R^{2}$ & & & & 0.1097 \\
\hline
\end{tabular}

Source: calcul de l'auteur à partir des données des enquêtes MICS 2000 et 2006

Nos résultats indiquent que le coefficient du terme d'interaction est négatif, ce qui indique que le conflit de 2002 a eu un effet négatif sur le recours à l'accouchement assisté des ménages vivant en zone CNO. Nous constatons qu'avec l'introduction des variables de contrôle l'effet du conflit est moins prononcé. Ce qui indique que les variables de contrôle permettent de réduire tout biais qui serait susceptible de surestimer nos estimations.

Si nous prenons le niveau d'éducation, comparativement aux ménages sans instruction, les ménages ayant le niveau primaire, secondaire et plus ont eu plus de chance d'être assisté à l'accouchement. L'éducation détermine donc la capacité de réaction en temps de crise.

Si nous considérons la religion, comparativement aux catholiques, ce sont les musulmanes qui ont eu plus de chance d'être assisté à l'accouchement. Cela pourrait s'expliquer par le fait que ces femmes ayant un groupe ethnique généralement proche des individus qui contrôlaient la zone $\mathrm{CNO}$ au cours du conflit de 2002 auraient certainement bénéficié de certaines facilités quant à l'accessibilité aux services de soins de santé. 


\section{Résultats et Discussion Pour le Conflit de 2010}

Pour le conflit de 2010, nous effectuons deux régressions. Dans la régression1, nous prenons comme traitél la zone CNO et comme contrôle la zone Centre-est. Le Tableau 4 présente les résultats de l'effet du conflit de 2010 sur le recours à l'accouchement assisté pour les traités 1 .

Tableau 4. Effet moyen du conflit de 2010 sur le recours à l'accouchement assisté

\begin{tabular}{|c|c|c|c|c|}
\hline Variables & \multirow[b]{2}{*}{ Coef. } & \multirow{2}{*}{$\begin{array}{c}\text { Bootstrap } \\
\text { Std.Err }\end{array}$} & \multirow[b]{2}{*}{$\rho>|Z|$} & \multirow[b]{2}{*}{ Effets } \\
\hline Accou_assis & & & & \\
\hline & \multicolumn{4}{|c|}{ Sans variables de contrôle } \\
\hline Traité1* year & -0.695 & 0.083 & $0.000 * * *$ & - \\
\hline & \multicolumn{4}{|c|}{ Avec variables de contre } \\
\hline Traité1*year & -0.514 & 0.109 & $0.000 * * *$ & - \\
\hline year_2012 & 0.426 & 0.135 & $0.002 * * *$ & + \\
\hline $\begin{array}{c}\text { Sexe_chef (ref. } \\
\text { male) }\end{array}$ & 0.900 & 0.111 & 0.419 & \\
\hline $\begin{array}{c}\text { Age_chef } \\
\text { (ref.adulte) }\end{array}$ & 0.106 & 0.888 & 0.906 & \\
\hline marital & -0.104 & 0.118 & 0.930 & \\
\hline Taille_men & -0.140 & 0.009 & 0.123 & \\
\hline \multicolumn{5}{|c|}{ Education (ref.no educ) } \\
\hline Primary & 0.366 & 0.761 & $0.000 * * *$ & + \\
\hline $\begin{array}{c}\text { Secondary \& } \\
\text { higher }\end{array}$ & 1.069 & 0.149 & $0.000 * * *$ & + \\
\hline \multicolumn{5}{|c|}{ Religion (ref. catholic) } \\
\hline Protestant & -0.233 & 0.130 & $0.075^{*}$ & + \\
\hline Muslim & 0.456 & 0.121 & $0.000 * * *$ & + \\
\hline Animist \& other & -0.802 & 0.137 & $0.000 * * *$ & - \\
\hline Cons & 0.215 & 0.211 & 0.307 & \\
\hline Observations & & & & 3018 \\
\hline Pseudo $R^{2}$ & & & & 0.0562 \\
\hline
\end{tabular}

Source : calcul de l'auteur à partir des données des enquêtes MICS 2006 et EDSCI 2012

Le Tableau 4 montre que le terme d'interaction est négatif pour les traités1, c'est-à-dire les ménages vivant en zone CNO.

En effet cette zone présentait une situation bien inquiétante avant les élections et le conflit de 2010 a amplifié les difficultés qui existaient déjà.

Pour la variable éducation, tout comme pour la crise de 2002, en 2010, comparativement aux ménages sans instruction, ceux ayant le niveau primaire, secondaire et plus, ont eu plus de chance d'être assisté à l'accouchement. Ce qui confirme une fois de plus que le niveau d'éducation est déterminant dans les prises de décisions pendant une crise socio-politique.

Au niveau de la religion, tout comme au cours de la crise de 2002, les musulmanes ont eu plus de chance d'être assisté à l'accouchement. 
Ensuite, nous effectuons la régression 2, en prenant comme traité 2 la zone Sud \& Abidjan et comme contrôle la zone Centre-est. Les résultats de l'effet moyen du conflit de 2010 sur le recours à l'accouchement assisté pour les traités 2 sont consignés dans le Tableau 5.

Tableau 5. Effet moyen du conflit de 2010 sur le recours à l'accouchement assisté

\begin{tabular}{|c|c|c|c|c|}
\hline Variables & & Bootstrap & & \\
\hline \multirow[t]{2}{*}{ Accou_assis } & Coef. & Std.Err & $\rho>|Z|$ & Effets \\
\hline & \multicolumn{4}{|c|}{ Sans variables de contrôle } \\
\hline \multirow[t]{2}{*}{ Traité1* year } & 0.582 & 0.093 & $0.000 * * *$ & + \\
\hline & \multicolumn{4}{|c|}{ Avec variables de contrôle } \\
\hline Traité1*year & 0.628 & 0.101 & $0.000 * * *$ & + \\
\hline year_2012 & -0.313 & 0.156 & 0.841 & \\
\hline $\begin{array}{c}\text { Sexe_chef (ref. } \\
\text { male) }\end{array}$ & -0.206 & 0.145 & 0.155 & \\
\hline $\begin{array}{l}\text { Age_chef } \\
\text { (ref.adulte) }\end{array}$ & 0.210 & 0.104 & $0.043^{*}$ & + \\
\hline $\begin{array}{c}\text { Marital (ref.no } \\
\text { married) }\end{array}$ & 0.151 & 0.122 & 0.216 & \\
\hline Taille_men & -0.031 & 0.009 & $0.002 * * *$ & - \\
\hline \multicolumn{5}{|c|}{ Education (ref.no educ) } \\
\hline Primary & 0.391 & 0.098 & $0.000 * * *$ & + \\
\hline Secondary \& higher & 1.301 & 0.183 & $0.000 * * *$ & + \\
\hline \multicolumn{5}{|c|}{ Religion (ref. catholic) } \\
\hline Protestant & 0.136 & 0.152 & 0.371 & \\
\hline Muslim & 0.309 & 0.143 & 0.031 & + \\
\hline Animist \& other & -0.607 & 0.178 & $0.001 * * *$ & - \\
\hline _Cons & 0.736 & 0.278 & 0.008 & + \\
\hline Observations & & & & 3018 \\
\hline Pseudo $R^{2}$ & & & & 0.0562 \\
\hline
\end{tabular}

Source: calcul de l'auteur à partir des données des enquêtes MICS 2006 et EDSCI 2012

Le Tableau 5 montre que le terme d'interaction est positif pour les traités2, c'est-à-dire les ménages vivant en zone Sud \& Abidjan. Ici, avec l'introduction des variables de contrôle l'effet du conflit de 2010 est plus prononcé. Les variables de contrôle ont donc contribué à réduire tout biais qui serait susceptible de sous-estimer nos estimations. Le signe positif observé indique que le conflit de 2010 a eu un effet positif sur le recours à l'accouchement assisté des ménages vivant en zone Sud \& Abidjan. Cela pourrait s'expliquer par le fait que l'Etat a renforcé sa politique en matière de prise en charge de l'accouchement assisté dans la zone pro-gouvernementale à cause des mouvements migratoires des populations de la zone CNO vers la zone Sud \& Abidjan en 2002. Les femmes vivant dans cette zone ont donc bénéficié des soins d'accouchement gratuit. Il faut aussi noter qu'étant donné 
que la crise de 2010 a été de courte durée de novembre 2010 à mars 2011, elle n'a donc pas désorganisé les structures mises en place.

\section{Conclusion}

En guise de conclusion nous pouvons dire que les deux conflits ont eu un impact négatif sur le recours à l'accouchement assisté en zone CNO et celui de 2010 a eu un impact positif en zone Sud \& Abidjan. Si nous comparons nos résultats à d'autres études, nos résultats obtenus en zone CNO sont semblables à ceux de Gudrun et al. (2015), qui indiquent que l'intensité des conflits réduit considérablement la probabilité qu'une femme donne naissance dans un établissement médical. Nous notons qu'il est capital de s'intéresser à la santé maternelle surtout à l'accouchement assisté dans un centre de santé en ce sens que le risque de mortalité est plus élevé durant le travail de l'accouchement, à la naissance et pendant la première semaine de vie. En collaboration avec les organismes internationaux, l'Etat ivoirien devrait garantir à chaque mère et à chaque nouveau-né l'accès à des soins de qualité et encore plus lorsque que celles-ci se trouvent dans des zones touchées par les conflits armés. "La santé pour tous" doit être assurée même en situation de crise et post-crise.

Comme perspectives des études pourraient être menées en vue d'analyser l'effet moyen des conflits armés de 2002 et de 2010 dans le domaine de l'emploi notamment au niveau primaire, secondaire et tertiaire. Outre, la méthode paramétrique une méthode non paramétrique pourrait être mobilisée notamment l'estimateur de l'effet moyen du traitement sur les traités ATT connu sous le vocable anglais de Average Treatment Effect on the Treated.

\section{References:}

1. Ahouré, A. \& Ouattara, W. (2009, septembre). Méthodes d'évaluation des programmes de

2. développement. Communication présentée à l'atelier de formation des responsables de la programmation et du suivi et évaluation des administrations publiques et privées, Grand-Bassam, Côte d'Ivoire.

3. Akresh R. (2016). Climate change, conflit, and children. Afrique contemporaine, 255,13-120.

4. Angrist, J., Imbens, G., \& Rubin D. (1996). Identification of causal effects using instrumental variables. Journal of the American Statistical Association, 91(434), 444-472.

5. Audibert, M. et al. (2015).Impact des plateformes multifonctionnelles sur l'activité économique des femmes et l'éducation des enfants au Mali. Etudes et Documents, $\mathrm{n}^{\circ} 12$, CE. Repéré à http://cerdi.org/production/show/id/1688/type_productio_id/1 
6. Becker, O. \& Ichino, A. (2002). Estimation of average treatment effects based on propensity scores. The Stata Journal, 2 (4), 358-377.

7. Blundell, R. \& Costa Dias, M. (2002). Alternative Approaches to Evaluation in Empirical Microeconomics. The Institute for Fiscal Studies Department of Economics, UCL cemmap working paper CWP10/02, 40 P.

8. Cadot, O., Fernades A., Gourdon, J., Mattoo A. (2014). Evaluating Aid for trade: A survey. The World Economy, 37 (4), 515-541.

9. Collier, P. \& Hoeffler A. (1998). On the Economic Causes of Civil War. Oxford Economic Papers, 50 (4), 563-595.

10. Collier, P. Hoeffler A., \& Söderbom (2004). On the duration of civil war. Journal of peace research, 41(3), 253-273.

11. Enquête Démographique et de Santé et à Indicateurs Multiples (EDSCI 2012). Côte d'Ivoire.

12. Fearon, James D. \& Davi, Laitin, D. (2003). Ethnicity, Insurgency, and Civil War. American Political Science Review. 97(1), 75-90.

13. Garcia, J. \& Bartlett, A. (2015). Occupations under fire : the labour market in a complex emergency. Oxford Economic Papers, 67(3): 687-714.

14. Givord, P. (2010). Méthodes économétriques pour l'évaluation de politiques publiques. Document de travail INSEE, 80 pages.

15. Heckman, J., Ichimura, H., Todd, P. (1998). Matching as an Econometric Estimator. Review of Economic Studies, 65, 261-294.

16. Hugon, P. (2001) . L'économie des conflits en Afrique. Revue internationale et stratégique, 43(3), 152-169. doi : 10.3917/ris.043.0152

17. Idrissa O. (2014). Essays on Education and Family Planning. (Thèse de doctorat). Université de Montréal, Canada.

18. Imbens, G. \& Wooldrige, J. (2009). Recent Developments in the Econometrics of Impact

19. Evaluation. Journal of Economic Literature, 47 (1), 5-86.

20. Institut National de la Statistique - Ministère du Plan et du Développement, Enquête Santé Mère et Enfant, 2010, Côte d'Ivoire.

21. Kaplan, R. (1994). The Coming Anarchy: how scarcity, crime, overpopulation and disease are threatening the social fabric of our planet. Atlantic Monthly, 273(2, février), 44-76.

22. Lecoq, A, Ammi, M., \& Bellarde, E. (2014). Le score de propension: un guide méthodologique pour les recherches expérimentales et quasiexpérimentales en education. Mesure et évaluation en education, 372 (2), 69-100. DOI: 10.7202/103591ar

23. Justino, P. (2012). War and poverty. Handbook of Economics of Peace and Security, Oxford University Press. 
24. Marbot, C. (2011).Une évaluation de la réduction d'impôt pour l'emploi de salariés à domicile. Institut National de la Statistique et des Etudes Economiques (INSEE), 42P.

25. Minoiu, C., \& Shemyakina O. (2014). Armed conflict, household victimization, and child health in Côte d'Ivoire. Journal Development Economic. 108, 237-255.

26. Nations Unies. (2015). Rapport 2015 : Objectifs du millénaire pour le développement, rapport du Secrétaire Général de l'ONU (Rapport final $n^{\circ} 15-04513$ ISBN 978-92-1-057475-4).

27. O.Hare, B. \& Southall, P. (2007). First do no harm : the impact of recent armed conflict on maternal and child health in Sub-saharan Africa. Journal of the Royal Society of Medecine 100 (12), 564-570.

28. Organisation Mondiale de la Santé (2015). Rapport mondial 2015 : Tendances de la mortalité maternelle: 1990-2015 (Rapport final $\left.\mathrm{n}^{\circ} 15.23\right)$.

29. Palmer et al. (2016). The long-term impact of war on health. HiCN Working paper 2016.

30. Perspective Monde (1999, décembre 24). Renversement du president Henri Konan Bédié en Côte d'Ivoire. Actualité en Côte d'Ivoire.

31. Perspective Monde (2002, septembre 19). Tentative de coup d'Etat contre le president Gbagbo. Actualité en Côte d'Ivoire.

32. Price, JI. \& Bohara, AK. (2013). Maternal health care amid political unrest: the effect of armed conflict on antenatal care utilization in Nepal. Health Policy Plan, 28(3), 309-319.

33. PRIO, The Peace Research Institue Olso. Armed conflict and maternal health care micro-level evidence from Sub-Saharan Africa: the Annual Convention of the American Political Science Association, 36 september 2015, San Francisco.

34. Ravallion, M. (2009). Do Poorer Countries Have Less Capacity for Redistribution? Policy Research Working Paper, World Bank, Washington, $\mathrm{DC}^{\circ} 5046$.

35. Rapachi B. (1994). Une introduction au bootstrap. Centre Interuniversitaire de calcul de Grenoble. 15 décembre 1994.

36. Rosembaum, P. \& Rubin, D. (1983). The central role of propensity score in observational studies for causal effects. Biometrika, 70 (1), 41-55.

37. Rubin, D (1974). Estimating Causal Effects of Treatments in Randomized and non-randomized Studies. Journal of Educational Psychology, 66 (5), 688-701. 
38. Save the Children (2014). La situation des mères dans le monde 2014: Sauver les mères et les enfants en situation de crise humanitaire. Repéré à www.savethechildren.org

39. Shemyakina, O. \& Minoiu, C. (2014). Armed conflict, household victimization and child health in Côte d'Ivoire. Journal of Development Economics, 108, 237-255. DOI: http://dx.doi.org/10.1016/j.jdeveco.2014.03.003

40. Tiembre, I. et al. (2011). Impact du conflit armé sur le système de santé d'un district sanitaire en Côte d'Ivoire. UFR des sciences médicales, Université d'Abidjan, Côte d'Ivoire et Département de la Santé de Séguéla, Côte d'Ivoire.

41. Yabilé, K. (2013). Impact du conflit armé sur l'accentuation de la pauvreté en Côte d'Ivoire. European Scientific Journal, 9 (8), ISSN : 1857. 


\section{ANNEXE}

Tableau 6.Nombre de victimes fatales

Pôle de développement Nombre de victimes fatales

\begin{tabular}{ccc} 
Pôle de développement & \multicolumn{2}{c}{ Nombre de victimes fatales } \\
& $2002 / 2003$ & $2010 / 2011$ \\
Centre & 2 & 14 \\
Centre-nord & 96 & 0 \\
Centre-ouest & 180 & 32 \\
Nord & 1 & 0 \\
Nord-est & 5 & 0 \\
Nord-ouest & 0 & 0 \\
Ouest & 890 & 401 \\
Sud & 77 & 283 \\
Sud-ouest & 25 & 0 \\
Centre-est & 0 & 0 \\
Total & 1276 & 742
\end{tabular}

Carte 1. Partition de la Côte d'Ivoire

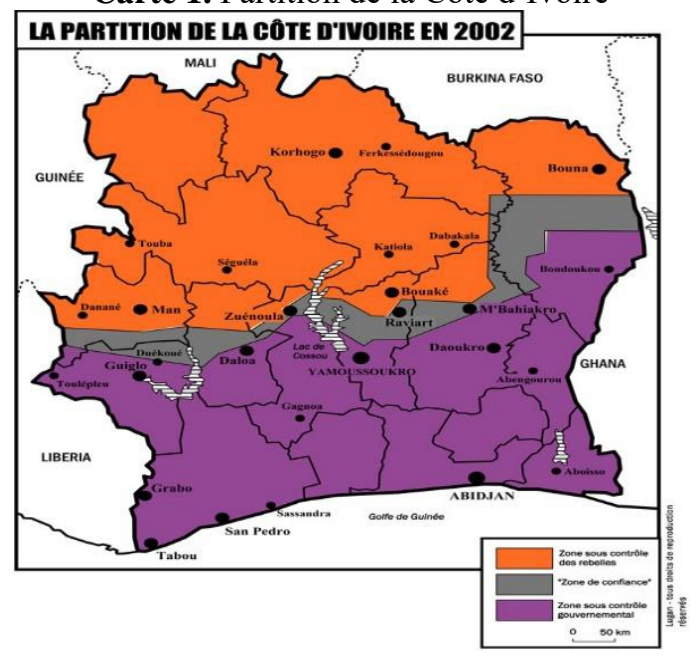

Source : ACLED 2002/2003 et ACLED 2010/2011 